\title{
Aplicativo para promoção da saúde ocupacional de profissionais da saúde em um
}

\section{hospital universitário}

\author{
Application to promote the occupational health of health professionals in a university hospital \\ Aplicación para promover la salud ocupacional de los profesionales de la salud en un hospital \\ universitário
}

Recebido: 12/07/2021 | Revisado: 16/07/2021 | Aceito: 19/07/2021 | Publicado: 27/07/2021

\author{
Jemima Silva Inocêncio \\ ORCID: https://orcid.org/0000-0002-3123-1755 \\ Universidade Federal de Sergipe, Brasil \\ E-mail: jemima-inocencio@ hotmail.com \\ Daniel Silva de Madureira \\ ORCID: https://orcid.org/0000-0002-2149-7891 \\ Instituto Federal de Sergipe, Brasil \\ E-mail: danielmadsv@gmail.com \\ Simone de Cássia Silva \\ ORCID: https://orcid.org/0000-0001-6076-9071 \\ Universidade Federal de Sergipe, Brasil \\ E-mail: scassia@gmail.com
}

\begin{abstract}
Resumo
O objetivo deste trabalho foi descrever o desenvolvimento de um aplicativo móvel para promoção da saúde ocupacional de profissionais da saúde em um hospital universitário. Trata-se de um estudo metodológico com interface participativa, de produção tecnológica, realizado a partir das etapas de elaboração textual do material disponível, mapeamento dos requisitos, prototipagem das telas do sistema e desenvolvimento da primeira versão. O aplicativo By Your Side (BYS) é uma ferramenta de promoção da saúde ocupacional, disponível gratuitamente para Android e iOS. Dentre as atividades propostas, destacam-se as orientações nutricionais, exercícios físicos, técnicas de relaxamento, cuidados com a saúde mental, orientações sobre o atendimento com profissionais, divulgação de programas da instituição, alertas de medicamentos, calculadoras para o Índice de Massa Corporal (IMC) e ingestão de água, entretenimento, diário de anotações e fórum com grupos e salas. O aplicativo disponibiliza os locais, dias disponíveis e fluxos dos atendimentos com profissionais para facilitar o acesso e adesão do usuário. Conclui-se que o BYS tem o potencial de ajudar os profissionais da área da saúde, promovendo uma vida mais saudável, aliviando a carga de estresse do trabalho, por meio de diversas atividades relacionadas a saúde física e mental.
\end{abstract}

Palavras-chave: Tecnologias e aplicativos de software; Profissionais de saúde; Saúde ocupacional.

\begin{abstract}
The objective of this work was to describe the development of a mobile application to promote the occupational health of health professionals in a university hospital. This is a methodological study with a participatory interface, technological production, carried out from the steps of textual elaboration of the available material, mapping of requirements, and prototyping of system screens and development of the first version. The By Your Side (BYS) application is an occupational health promotion tool, available free for Android and iOS. Among the proposed activities, nutritional guidance, exercise, relaxation techniques, mental health care, guidance on professional assistance, dissemination of programs of the institution, medication alerts, and calculators for the Mass Index stand out. Body (BMI) and water intake, entertainment, journaling and forum with groups and rooms. The application makes available the locations, available days and service flows with professionals to facilitate user access and adherence. It is concluded that BYS has the potential to help health professionals, promoting a healthier life, relieving the work stress load, through various activities related to physical and mental health.
\end{abstract}

Keywords: Technologies and software applications; Health professionals; Occupational health.

\section{Resumen}

El objetivo de este trabajo fue describir el desarrollo de una aplicación móvil para promover la salud ocupacional de los profesionales de la salud en un hospital universitario. Se trata de estudio metodológico con interfaz participativa, producción tecnológica, realizado a partir de los pasos de elaboración textual del material disponible, mapeo de requerimientos, prototipado de pantallas del sistema y desarrollo de la primera versión. By Your Side (BYS) es una herramienta de promoción de la salud ocupacional, disponible de forma gratuita para Android e iOS. Entre las 
actividades propuestas, se destacan orientación nutricional, ejercicio, técnicas de relajación, atención en salud mental, orientación sobre asistencia profesional, difusión de programas de la institución, alertas de medicación, calculadoras para el Índice de Masa. Cuerpo (IMC) e ingesta de agua, entretenimiento, diario y foro con grupos y salas. La aplicación pone a disposición las ubicaciones, los días disponibles y los flujos de servicio con los profesionales para facilitar el acceso y adherencia de los usuarios. Se concluye que BYS tiene el potencial de ayudar a los profesionales de la salud, promoviendo una vida más saludable, aliviando la carga de estrés laboral, a través de diversas actividades relacionadas con la salud física y mental.

Palabras clave: Tecnologías y aplicaciones de software; Profesionales de la salud; Salud ocupacional.

\section{Introdução}

A inovação tecnológica representa um processo dinâmico de criação de novos produtos, ideias ou métodos de fabricação, fundamental no que diz respeito ao crescimento no atual mundo globalizado. Também pode ser entendida como a introdução de melhorias significativas em produtos ou processos existentes (Fuck \& Vilha, 2012). Considera-se uma inovação tecnológica quando o produto ou serviço produz soluções de problemas ou mudanças no campo científico, acadêmico ou na prática, devendo estar aliada às necessidades das pessoas (Ferreira et al., 2021; Fuck \& Vilha, 2012).

Com a criação do Sistema Único de Saúde (SUS), nota-se um crescimento e desenvolvimento nos processos de inovação tecnológica em saúde nos últimos anos (Felipe et al., 2019). Essas novas tecnologias envolvem a produção de medicamentos e equipamentos, elaboração de sistemas e criação de programas e protocolos assistenciais (Lorenzetti et al., 2012).

Nesse processo, a enfermagem destaca-se pela evolução na produção de novas tecnologias para sua prática e seu objeto de trabalho no sentido de melhorar a saúde da população (Erdmann, 2013; Goyatá et al., 2012; Gaidzinski et al., 2009). Ademais, as inovações tecnológicas na enfermagem buscam impactar na qualidade de vida e saúde dos profissionais da área, visto que estes são expostos a cargas físicas, psíquicas e ergonômicas dentro do contexto ocupacional (Oliveira et al., 2009). Com a pandemia do Coronavírus, os profissionais da saúde sofreram agravos psíquicos necessitando de estratégias de cuidados em saúde mental, como a criação de aplicativos móveis (Saidel, 2020).

A produção de aplicativos no processo de trabalho surgiu como uma inovação tecnológica em saúde compartilhada por diferentes estudos (Barra et al., 2017; Santos, 2010; Silva et al., 2018), apesar de a maioria não serem divulgada no meio científico (Ferreira et al., 2021). A partir desta perspectiva, surgiu a iniciativa do presente estudo, o qual teve como proposta utilizar a inovação tecnológica como uma ferramenta para cuidar da saúde ocupacional de trabalhadores da saúde, visto que este grupo foi bastante afetado durante o período da pandemia da COVID-19 (Ramos-Toescher et al., 2020). Dessa forma, este estudo objetivou descrever o desenvolvimento de um aplicativo móvel para promoção da saúde ocupacional de profissionais da saúde em um hospital universitário.

\section{Metodologia}

Trata-se de um estudo metodológico, qualitativo, de natureza aplicada na versão de produção tecnológica, que tem como eixo focal o desenvolvimento, avaliação e aperfeiçoamento de instrumentos e estratégias metodológicas (POLIT et al., 2004). O aplicativo, denominado BYS (By Your Side), foi desenvolvido no período de julho de 2020 a abril de 2021, para os profissionais da saúde do hospital universitário da Universidade Federal de Sergipe (HU-UFS), localizado em Aracaju/SE.

Para a construção do aplicativo como estratégia de inovação tecnológica, atribuíram-se as seguintes etapas:

a) Elaboração textual do material disponível no aplicativo em parceria com a equipe multiprofissional do HU-UFS das áreas de Nutrição, Enfermagem, Psicologia, Psiquiatria, Educação Física, Fisioterapia e Terapia Ocupacional;

b) Mapeamento dos requisitos para construção da ferramenta com suas funcionalidades e técnicas inseridas no sistema;

c) Prototipagem das telas do sistema; 
d) Desenvolvimento da primeira versão do sistema, a qual inclui a emissão de relatórios gerenciais para a gestão dos encaminhamentos de funcionários aos especialistas, número de usuários e acessos diários, downloads de livros, cartilhas e materiais educativos da instituição, assim como acesso às informações referentes a todas as funções do aplicativo.

O protótipo do aplicativo foi desenvolvido através da linguagem JavaScript, utilizando-se do ambiente de execução Node.JS (Runtime Environment), o qual faz uso do interpretador V8, desenvolvido pelo Google. Para a criação na plataforma Android, considerada como base para o desenvolvimento do protótipo, utilizou-se do Android Studio, que corresponde a um Ambiente de Desenvolvimento Integrado - IDE (Integrated Development Enviroment). Utilizou-se também um framework React-Native 0.63.2 para criação de aplicativos para aparelhos móveis e conversão para diversas plataformas (Windows, Mac, Linux, Android, iOS, BlackBerry e HTML5) após finalizado o protótipo na Android.

A administração do aplicativo é feita através do site https://portal.bys-app.com/login. Nesse site, o administrador atualiza o aplicativo e todas as publicações, aceita usuários como administradores e faz a moderação de todos os grupos, salas de conversas e comentários. A tecnologia utilizada na criação do portal foi o Vue.js 2.6.12 que significa um framework progressivo do JavaScript de código aberto (open source).

\section{Resultados e Discussão}

O BYS é um aplicativo de saúde ocupacional e trata-se de uma produção tecnológica gratuita disponível para Android e iOS destinado aos profissionais de saúde do HU-UFS. O processo de produção foi baseado na engenharia de software usando o conceito de prototipagem de acordo com as etapas de reconhecimento do problema, pesquisa de viabilidade, análise, projeto, implementação, testes e manutenção (Page-Jones, 1988). No sistema de produção do aplicativo foi utilizada uma técnica chamada Mapeamento Objeto-Relacional (ORM) a fim de reduzir as necessidades e escrever códigos de conexão e linguagens de consulta estruturada (SQL).

O aplicativo é uma ferramenta para uma vida mais saudável, utilizando-se da tecnologia a seu favor por meio de diversas atividades. Após a realização do cadastro e login (Figura 1), o usuário tem acesso ao conteúdo da plataforma. 
Figura 1 - Exibição da tela de cadastro e login no aplicativo móvel BYS, Aracaju - SE, 2021.

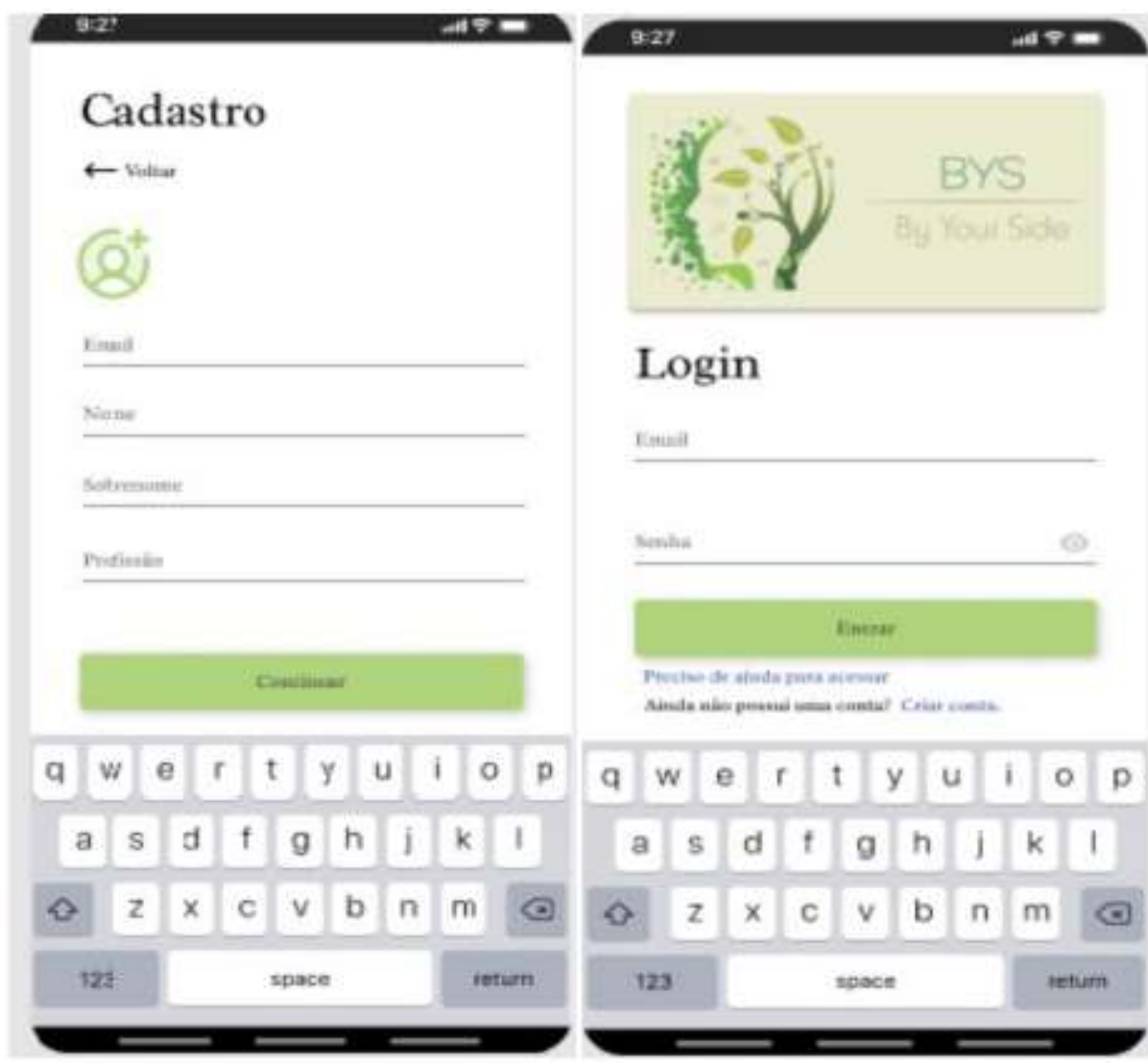

Fonte: Autores.

Quando se trata de saúde ocupacional, os profissionais da saúde possuem destaque pela constante carga psíquica que carregam em suas atividades laborais (Llapa-Rodriguez et al., 2018). Assim, a saúde mental destes trabalhadores merece atenção, especialmente no contexto específico da pandemia, a partir de intervenções direcionadas (Saidel et al., 2020). O desenvolvimento do aplicativo foi uma ferramenta inovadora que buscou promover o autocuidado, de forma holística, entre os profissionais a partir das suas necessidades.

Dentre as atividades propostas pelo aplicativo, destacam-se as orientações nutricionais, exercícios físicos, técnicas de relaxamento, cuidados com a saúde mental e orientações sobre o plantão psicológico do próprio serviço (Figura 2). Além disso, o BYS disponibiliza a divulgação de projetos e programas da instituição relacionados à humanização e saúde mental do trabalhador, a exemplo das atividades de Práticas Integrativas e Complementares em Saúde para os funcionários através de aulas on-line e incentivo à prática de exercícios físicos, como grupos de corrida e capoeira. 
Figura 2 - Exibição da tela do Feed do aplicativo com as atividades propostas ao usuário, Aracaju - SE, 2021.

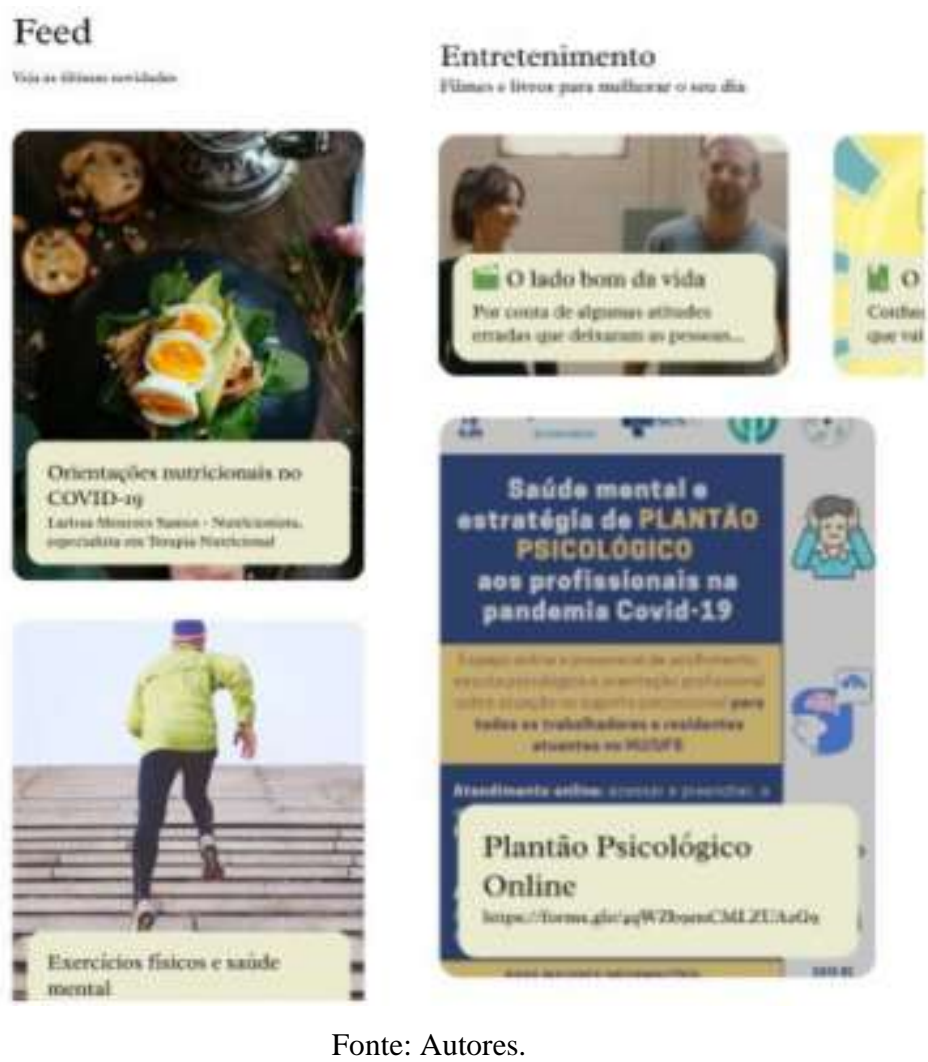

As variedades de ações inseridas no aplicativo ocorreram em parceria com especialistas de diversas áreas. Por meio desta capilaridade, busca-se estimular a participação dos colaboradores a se tornarem os facilitadores do seu próprio cuidado à saúde com vistas ao equilíbrio físico, mental e emocional. Incluiu-se também um espaço para dicas de filmes, atividades de lazer e downloads de e-books, frases motivacionais e alertas de acordo com os horários das medicações em uso (Figura 3). 
Research, Society and Development, v. 10, n. 9, e33010918164, 2021

(CC BY 4.0) | ISSN 2525-3409 | DOI: http://dx.doi.org/10.33448/rsd-v10i9.18164

Figura 3 - Exibição da tela de alertas de medicamentos do usuário, Aracaju - SE, 2021.

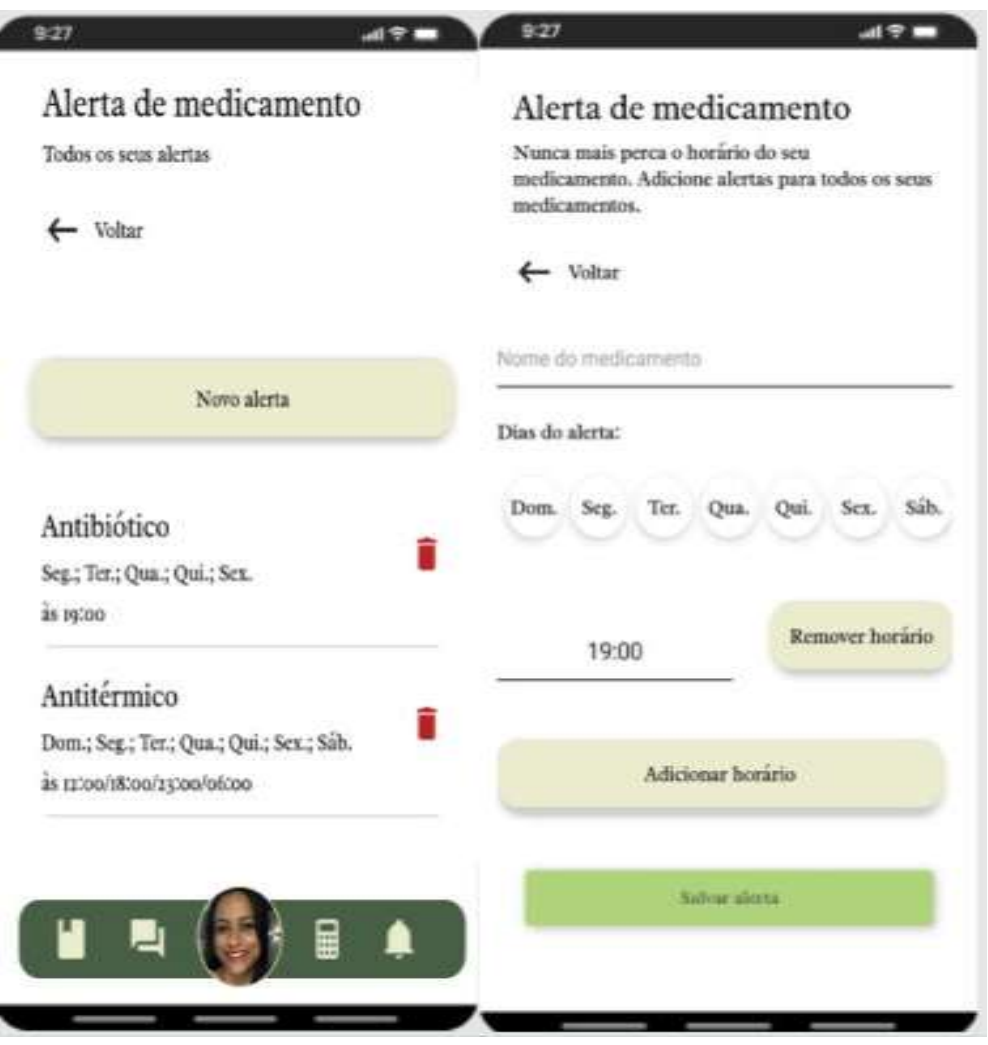

Fonte: Autores.

Um dos motivos que contribuem na falta de adesão medicamentosa é o esquecimento da administração das doses pelo indivíduo (Oliveira et al., 2020). Assim, para melhor comodidade, o usuário tem a opção de cadastrar mais de um medicamento para que o sistema emita um alarme no horário programado. Como as alterações no peso podem ter relação com os transtornos mentais (Melca \& Fortes, 2014), foram inseridas as funções para cálculo do Índice de Massa Corpóreo (IMC) e da quantidade ideal de ingestão diária de água (Figura 4). 
Research, Society and Development, v. 10, n. 9, e33010918164, 2021

(CC BY 4.0) | ISSN 2525-3409 | DOI: http://dx.doi.org/10.33448/rsd-v10i9.18164

Figura 4 - Exibição da tela das funções de cálculo do IMC e seu resultado, Aracaju - SE, 2021.

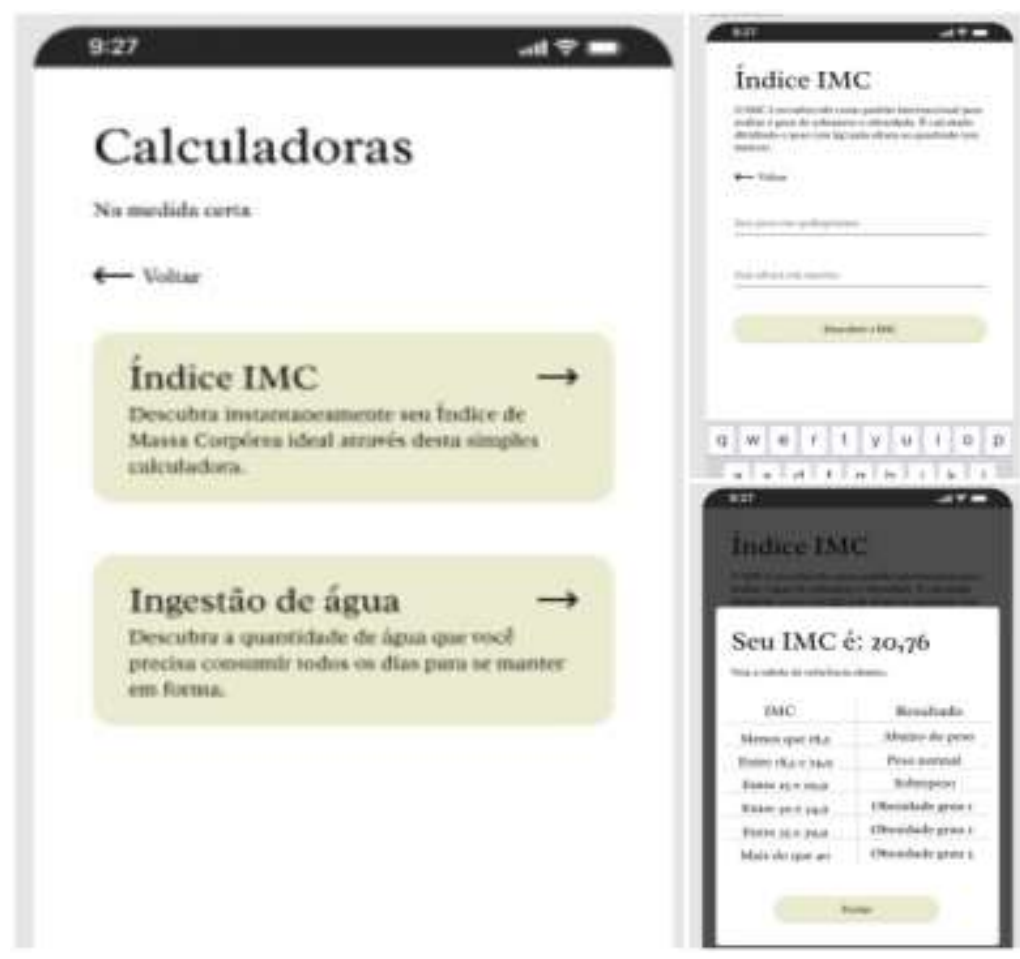

Fonte: Autores.

A escrita e o registro das emoções são recursos terapêuticos eficazes quando se busca aliviar as tensões e promover bem-estar (Benetti \& Oliveira, 2016). O usuário também pode registrar suas emoções através da escrita sobre seus sentimentos em diário, assim como as atitudes diante do que foi sentido (Figura 5). Com o passar do tempo ele terá um histórico de registro de pensamentos, o que pode contribuir para o entendimento do seu comportamento, constituindo uma opção efetiva para quem faz acompanhamento com terapeuta. 
Figura 5 - Exibição da tela do perfil, do diário e do acompanhamento da história do usuário, Aracaju - SE, 2021.

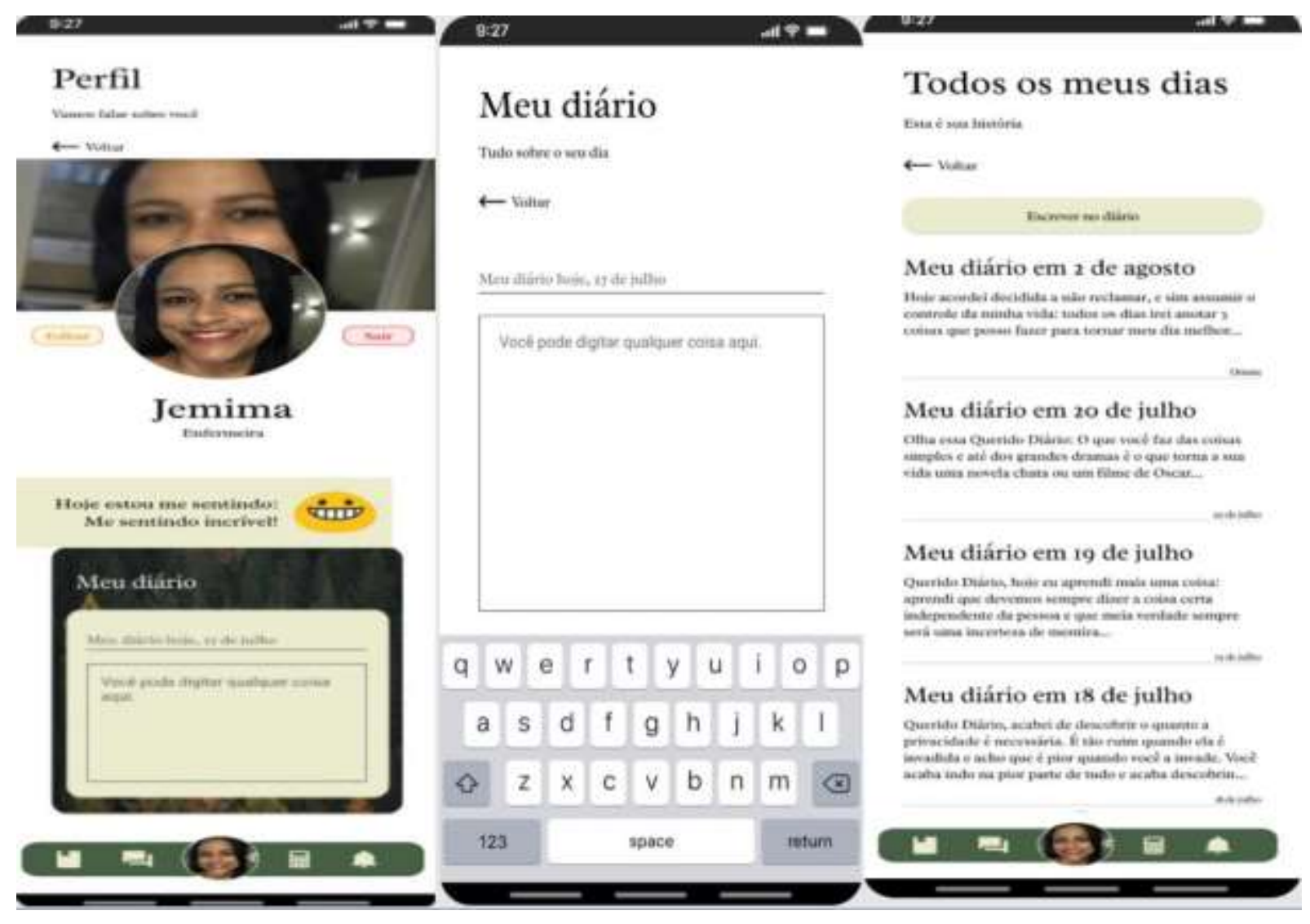

Fonte: Autores.

A inovação tecnológica causa impacto e transformações na saúde de seus usuários. Para isso, deve-se entender as necessidades dos indivíduos, sendo importante o apoio institucional para sua implementação (Ferreira et al., 2021). Dentro do campo promoção em saúde, a realidade virtual representa importante ferramenta educacional, visto que permite o entendimento do usuário com relação ao seu próprio estado de saúde (Campos Filho et al., 2020).

A partir da criação da ferramenta, observou-se boa adesão e participação dos profissionais com relação ao uso do aplicativo. O principal benefício será o acompanhamento periódico dos funcionários da instituição, oferecendo ao trabalhador mais segurança na relação de trabalho que reflete no melhor desempenho e motivação, visto que este é um fator importante para redução do absenteísmo (Furlan et al., 2018). Além disso, o cuidado através do desenvolvimento tecnológico aproxima a produção acadêmica do setor produtivo e da sociedade, contribuindo para as ações estratégicas do SUS.

Vale ressaltar que o aplicativo é um direcionamento sobre o autocuidado dos profissionais. Neste sentido, o acompanhamento terapêutico torna-se um recurso fundamental aos usuários que necessitarem, o qual deve ser realizado por um profissional especializado (Silva et al., 2021). Dessa forma, o aplicativo disponibiliza os locais, dias disponíveis e fluxos dos atendimentos para facilitar o acesso e adesão do usuário.

É importante destacar a lacuna nas publicações científicas sobre o desenvolvimento de aplicativos para o cuidado da saúde ocupacional dos profissionais da saúde. Destarte, em termos de registro na literatura, o aplicativo BYS pode ser considerado uma proposta inovadora. Como limitações deste trabalho, destaca-se que a etapa de validação não pôde ser realizada devido ao curto espaço de tempo, limitando este estudo à descrição do desenvolvimento do aplicativo. 


\section{Conclusão}

O aplicativo BYS é um produto de inovação tecnológica gratuita disponível aos profissionais da saúde. A ferramenta visa promover uma vida mais saudável, por meio de diversas atividades relacionadas a saúde física e mental.

Sugere-se, a partir desse trabalho, novos estudos acerca da avaliação do aplicativo quanto a sua funcionalidade e repercussão na vida dos profissionais, assim como sobre o desenvolvimento de aplicativos direcionados à saúde ocupacional dos trabalhadores da saúde.

\section{Referências}

Barra, D. C. C. et al. (2017). Métodos Para Desenvolvimento De Aplicativos Móveis Em Saúde: Revisão Integrativa Da Literatura. Texto Contexto Enferm, 26(4), e2260017.

Benetti, I. C. \& Oliveira, W. (2016). O poder terapêutico da escrita: quando o silêncio fala alto. Cadernos Brasileiros de Saúde Mental, 8(19), 67-77.

Campos Filho, A. S. et al. (2020). Realidade virtual como ferramenta educacional e assistencial na saúde: uma revisão integrativa. J. Health Inform, 12(2), 5863.

Erdmann, A. L. (2013). A inovação em enfermagem. Cienc. Enferm., 19(3), 7-9.

Felipe, M. S. S. et al. (2019). Um olhar sobre o Complexo Econômico Industrial da Saúde e a Pesquisa Translacional. Saúde debate, $43(123)$, $1181-93$.

Ferreira, D. S. et al. (2021). Aplicativo móvel para a práxis educativa de enfermeiros da estratégia saúde da família: ideação e prototipagem. Esc. Anna. Nery, 25(1), e20190329.

Fuck, M. P. \& Vilha, A. M. (2012). Inovação Tecnológica: da definição à ação. Revista de Artes e Humanidades, 9, 1-21.

Furlan, J. A. S. et al. (2018). O profissional de enfermagem e sua percepção sobre absenteísmo. Rev. Eletr. Enf., 20, 1-9.

Gaidzinski, R. et al. (2012). Dimensionamento informatizado de profissionais de Enfermagem: inovação tecnológica. Rev Esc Enferm USP, 43(2), 1314-9.

Goyatá, S. et al. (2012). Ensino do processo de enfermagem a graduandos com apoio de tecnologias da informática. Acta Paul Enferm, 25(2), 243-8.

Llapa-Rodriguez, E. O. et al. (2018). Estresse ocupacional em profissionais de enfermagem. Rev enferm UERJ, 26, e19404.

Lorenzetti, J. et al. (2012). Tecnologia, Inovação Tecnológica E Saúde: Uma Reflexão Necessária. Texto Contexto Enferm, 2(2), 432-9.

Melca, I. A. \& Fortes, S. (2014). Obesidade e transtornos mentais: construindo um cuidado efetivo. Revista Hospital Universitário Pedro Ernesto, 13(1), 1825 .

Oliveira, D. F. et al. (2020). Fatores Associados À Baixa Adesão Ao Tratamento Farmacológico De Pacientes Atendidos Por Um Centro Integrado De Saúde. Brazilian Journal of Natural Sciences, 3(3), 430-40.

Oliveira, J. A. S. et al. (2009). Riscos ocupacionais no contexto hospitalar: desafio para a saúde do trabalhador. Rev. salud pública, $11(6)$. 909-917.

Page-Jones, M. (1988). Projeto estruturado de sistemas. São Paulo: McGraw-Hill.

Polit, D. F. et al (2004). Fundamentos de Pesquisa em Enfermagem: métodos, avaliação e utilização. Porto Alegre: Artmed.

Ramos-Toescher, A. M. et al. (2020). Saúde mental de profissionais de enfermagem durante a pandemia de COVID-19: recursos de apoio. Esc Anna Nery, 24, e20200276.

Saidel, M. G. B. et al. (2020). Intervenções em saúde mental para profissionais de saúde frente a pandemia de Coronavírus. Rev enferm UERJ, 28 , e49923.

Santos, S. R. (2010). Informática em enfermagem: desenvolvimento de software livre com aplicação assistencial e gerencial. Rev Esc Enferm USP, 44(2), 295301 .

Silva, A. M. A. et al. (2018). Mobile technologies in the Nursing area. Rev Bras Enferm, 71(5), 2570-8.

Silva, J. L. L. et al. (2021). Aplicativo mentalpro para auxílio na suspeição de transtornos mentais na Atenção Básica em Saúde. Research, Society and Development, 10(7), e12110716318. 